\title{
Ppp1r1b-IncRNA inhibits PRC2 at myogenic regulatory genes to promote cardiac and skeletal muscle development in mouse and human
}

\author{
XUEDONG KANG ${ }^{1,2}$ YAN ZHAO, ${ }^{1,2}$ GLEN VAN ARSDELL, ${ }^{1,3}$ STANLEY F. NELSON, ${ }^{1,4,5,6}$ \\ and MARLIN TOUMA ${ }^{1,2,5,6,7,8,9}$ \\ ${ }^{1}$ Department of Pediatrics, David Geffen School of Medicine, University of California Los Angeles, Los Angeles, California 90095, USA \\ ${ }^{2}$ Neonatal/Congenital Heart Laboratory, Cardiovascular Research Laboratory, University of California Los Angeles, Los Angeles, California 90095, USA \\ ${ }^{3}$ Department of Cardiothoracic Surgery, David Geffen School of Medicine, University of California Los Angeles, Los Angeles, California 90095, USA \\ ${ }^{4}$ Department of Neurology, David Geffen School of Medicine, University of California Los Angeles, Los Angeles, California 90095, USA \\ ${ }^{5}$ Department of Human Genetics, Institute of Precision Health, David Geffen School of Medicine, University of California Los Angeles, Los \\ Angeles, California 90095, USA \\ ${ }^{6}$ Institute of Precision Health, David Geffen School of Medicine, University of California Los Angeles, Los Angeles, California 90095, USA \\ ${ }^{7}$ The Molecular Biology Institute, David Geffen School of Medicine, University of California Los Angeles, Los Angeles, California 90095, USA \\ ${ }^{8}$ Children's Discovery and Innovation Institute, Department of Pediatrics, David Geffen School of Medicine, University of California Los Angeles, \\ Los Angeles, California 90095, USA \\ ${ }^{9}$ Eli and Edythe Broad Stem Cell Institute, David Geffen School of Medicine, University of California Los Angeles, Los Angeles, California 90095, USA
}

\begin{abstract}
Long noncoding RNAs (IncRNAs) have emerged as critical epigenetic regulators and play important roles in cardiac development and congenital heart disease. In a previous study, we identified a novel IncRNA, Ppp1r1b, with expression highly correlated with myogenesis. However, the molecular mechanism that underlies Ppp1r1b-IncRNA function in myogenic regulation is unknown. By silencing Ppp1r1b-IncRNA, mouse C2C12 and human skeletal myoblasts failed to develop fully differentiated myotubes. Myogenic differentiation was also impaired in PPP1R1B-IncRNA deficient human-induced pluripotent stem cell-derived cardiomyocytes (hiPSCs-CMs). The expression of myogenic transcription factors, including MyoD, Myogenin, and Tbx5, as well as sarcomere proteins, was significantly suppressed in Ppp1r1b-IncRNA inhibited myoblast cells and neonatal mouse heart. Histone modification analysis revealed increased H3K27 tri-methylation at MyoD1 and Myogenin promoters in GapmeR treated C2C12 cells. Furthermore, Ppp1r1b-IncRNA was found to bind to Ezh2, and chromatin isolation by RNA purification (ChIRP) assay revealed enriched interaction of Ppp1r1b-IncRNA with Myod1 and Tbx5 promoters, suggesting that Ppp1 1 b-IncRNA induces transcription of myogenic transcription factors by interacting with the polycomb repressive complex 2 (PRC2) at the chromatin interface. Correspondingly, the silencing of Ppp1r1bIncRNA increased EZH2 binding at promoter regions of myogenic transcription factors. Therefore, our results suggest that Ppp1r1b-IncRNA promotes myogenic differentiation through competing for PRC2 binding with chromatin of myogenic master regulators during heart and skeletal muscle development.
\end{abstract}

Keywords: long noncoding RNA; H3K27me3; epigenome; myogenesis

\section{INTRODUCTION}

Long noncoding RNAs (IncRNAs), which comprise the bulk of the noncoding genome, have been proven to play

Abbreviations: LncRNA, long noncoding RNA; ChIRP, chromatin isolation by RNA purification; PRC2, polycomb repressive complex 2; RIP, RNA immunoprecipitation; CHIP, chromatin immunoprecipitation; HiPSCs, human-induced pluripotent stem cells; sk-Fmhc, fastskeletal myosin heavy chain; CHD, congenital heart defect

Corresponding author: mtouma@mednet.ucla.edu

Article is online at http://www.rnajournal.org/cgi/doi/10.1261/rna. 073692.119. Freely available online through the RNA Open Access option. important roles in maintaining cardiovascular system homeostasis (Ounzain et al. 2013; Touma et al. 2016). Therefore, exploring the mechanisms that underlie their function in heart development will further deepen our understanding of heart development and provide new insight for promoting translational applications.

Skeletal and cardiac muscle both arise from myogenic mesodermal lineages and share many characteristics. It

(C) 2020 Kang et al. This article, published in RNA, is available under a Creative Commons License (Attribution 4.0 International), as described at http://creativecommons.org/licenses/by/4.0/. 
was reported that the expression patterns of cardiac and skeletal muscle transcription factors and fast-skeletal myosin heavy chain (sk-fMHC) within ventricular myocardium and skeletal muscle were similar at embryonic day (ED) 20 , and the expression patterns became cardiac or skeletal muscle specific only during postnatal development (Clause et al. 2012). Some types of muscular dystrophies also associate with cardiomyopathy and chronic cardiac diseases (Muntoni 2003). The overlapping pattern of muscle-specific gene expression in cardiac and skeletal muscle and the common origin of cardiac and skeletal muscle pathologies suggest the existence of a common underlying regulatory scheme for the control of muscle genes expression, especially in their immature phase (Hassan et al. 2014).

Several myogenic transcription factors have been identified. Among them, the basic helix-loop-helix (bHLH) family (MYOD1, MYF5, and MYOG) were discovered to be key regulators of muscle development (Hernández-Hernández et al. 2017). Likewise, heart development is governed by a core set of transcription factors (NKX2.5, MEF2, GATA, TBX, and Hand) (Garry and Olson 2006). However, these known core set of transcription factors mainly controls the expansion of chamber myocardium but not the initial differentiation and commitment to cardiomyocytes (Garry and Olson 2006; Olson 2006). The precise molecular mechanisms of the initial differentiation are not entirely clear in heart development. Therefore, detailed understanding of the mechanisms of the initial differentiation is necessary, especially for cardiomyocyte regeneration.

Epigenetic modifications at the transcription factor binding sites play critical roles in the regulation of gene expression, including the myogenic differentiation genes (Kouzarides 2007). In embryonic stem cells or induced pluripotent stem cells (ESCs/iPSCs), H3K27me3 functions as an "epigenetic barrier" against ESC/iPSC differentiation. In the meantime, H3K27 demethylation facilitates MyoD1-mediated myogenic differentiation (Aloia et al. 2013; Adam and Fuchs 2016). During direct cardiac reprogramming, increased level of H3K27me3 at the promoter sites of cardiac transcription factors, such as Mef2c, Gata4, and Tbx5, corresponds to decrease of mRNA expression of cardiac genes (Liu et al. 2016).

LncRNAs may carry out both gene inhibition and gene activation through a range of diverse mechanisms. Targeted modulation of epigenetic modifiers, such as the Polycomb repressive complex (PRC) subunits, by IncRNAs has been suggested as a general mechanism for trans-acting IncRNA in gene regulation (Prasanth and Spector 2007). PRC-mediated gene silencing is mainly dependent on the EZH2 dependent H3K27 tri-methylation (Schuettengruber and Cavalli 2009). LncRNAs can directly modulate $\mathrm{EZH} 2$ activity or recruit $\mathrm{EZH} 2$ to the promoter region of genes to achieve targeted gene repression (Su et al. 2018). Moreover, IncRNAs can also serve as
EZH2 effectors or regulators (Su et al. 2018). For the inhibition of chromatin methylation, a model was suggested that the interactions of PRC2 with IncRNAs and chromatin are mutually antagonistic (Wang et al. 2017) and IncRNAs inhibit PRC2 methyltransferase activity by blocking PRC2 binding to targeted chromatin (Beltran et al. 2016).

In this report, we investigated the expression patterns of Ppp1r1b-IncRNA during myoblast differentiation, assessed the effect of down-regulation of Ppp1r1b-IncRNA on myogenesis in vitro and in vivo, and examined its impact on $\mathrm{H} 3 \mathrm{~K} 27$ methylation status at promoter regions of myogenic transcription factors, including MyoD, Myogenin, and Tbx5. We demonstrated interaction between Ppp1r1b-IncRNA, with Ezh2, and targeted promoters of myogenic transcription factors. These findings revealed that Ppp1r1b-IncRNA regulates myocyte differentiation by negatively modulating H3K27me3 of myogenic transcription factor genes via competing for PRC2 binding with chromatin.

\section{RESULTS}

\section{Ppp1r1b-IncRNA is overexpressed in differentiated C2C12 cells in association with myogenesis}

To determine the effects of Ppp1r1b-IncRNA on myogenesis, C2C12, an immortalized mouse myoblast cell line, was used. We cultured C2C12 cells in high serum conditions for rapid proliferation and low serum conditions for differentiation. The expression of Ppp1r1b-IncRNA in differentiated $\mathrm{C} 2 \mathrm{C} 12$ cells was significantly higher than that in the nondifferentiated cells (Fig. 1A). Subcellular localization of Ppp1r1b-IncRNA was characterized after subcellular fractionation of $\mathrm{C} 2 \mathrm{C} 12$ cells. Ppp1r1b-IncRNA was equivalently distributed between nucleus and cytoplasm in both nondifferentiated and differentiated cells (Fig. 1B).

In order to elucidate the functional role of Ppp1r1bIncRNA, we treated C2C12 cells cultured in differentiation media with $50 \mathrm{nM}$ antisense Ppp1 r1b-IncRNA GapmeR for $48 \mathrm{~h}$. The GapmeR treatment decreased Ppp1r1b-IncRNA expression by more than $70 \%$ in differentiated $\mathrm{C} 2 \mathrm{C} 12$ cells (Fig. 1C). Morphology of cells was visualized with a light microscope (Fig. 1D). A significant lower degree of differentiation was observed in the GapmeR treated cells that remained in a quiescent mono-nucleated status and did not differentiate into myotubes. These results indicate that Ppp1r1b-IncRNA expression is required for normal myogenesis.

\section{Human PPP1R1B-IncRNA exhibits similar roles in myogenesis of human myoblast}

LncRNAs are known to be poorly conserved across species. Furthermore, IncRNAs are less conserved at the primary nucleotide sequences compared with protein 

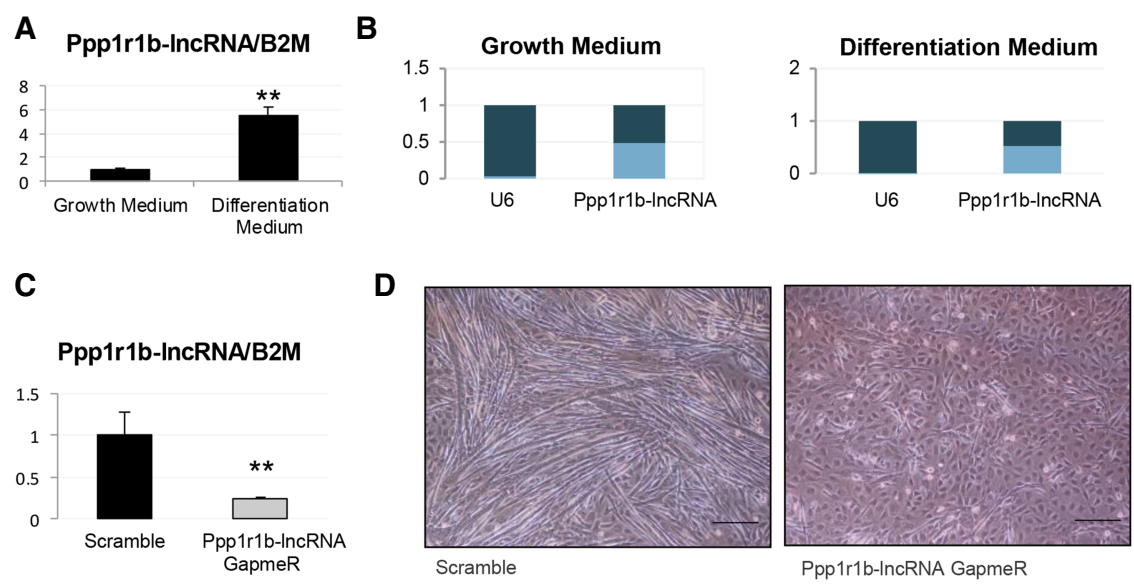

FIGURE 1. Ppp1r1b-IncRNA is induced during myogenesis and regulates $\mathrm{C} 2 \mathrm{C} 12$ myoblast differentiation. $(A, C)$ Quantitative real-time PCR analysis of Ppp1r1b-IncRNA expression after $2 \mathrm{~d}$ of differentiation $(A)$ and GapmeR treatment $(C)$. (B) Subcellular localization of Ppp1r1bIncRNA in myoblasts. U6 RNA was used as a nuclear location control. (D) Light microscope images depict the morphology of C2C12 cells after $2 \mathrm{~d}$ of differentiation and Ppp1r1b-GapmeR, or Scramble, treatment. Scale bar, $200 \mu \mathrm{m} . N=4$ biological replicates per condition; error bars, standard error of the mean; $\left(^{* *}\right) P<0.01$.

coding genes (Paralkar et al. 2014). It was reported that the repeat-containing regions in Xist IncRNA are generally unstructured and are functionally bound by protein cofactors (Smola et al. 2016). However, using comparative sequence, structural, and functional analyses, Karner et al. (2019) demonstrated that the functional conservation of IncRNA is independent from sequence and structural divergence (Karner et al. 2019). Therefore, determining functionally conserved and specific Ppp1r1b-IncRNA in human is essential. The human PPP1R1B locus has three noncoding transcripts. By comparing PPP1R1B-IncRNA transcripts between mouse and human, we identified a potential human ortholog of Ppp1r1b-IncRNA. However, unlike the mouse Ppp1r1b-IncRNA, in which no open reading frame (ORF) was detected, the human PPP1R1BIncRNA contained several short ORFs that can potentially produce small peptides. To examine the coding potential, we cloned the full-length mouse and human IncRNAs and carried out an in vitro expression and translation assay (Materials and Methods; Supplemental Fig. 1). Consistent with IncRNAs properties, no peptide was produced confirming the lack of a functional ORF in both mouse and human orthologs.

Like in mice, the human PPP1R1B-IncRNA was also induced during differentiation (Fig. 2A-C). To examine whether the human ortholog carries a similar function, we first used siRNAs directed against human PPP1R1BIncRNA in a human myoblast cell line and achieved efficient suppression in the nuclear and cytosolic compartments (Fig. 2D). Myotube differentiation was also impaired, as demonstrated by decreased myosin protein expression compared to control cells (Fig. 2A,B) and reduced fusion index (Fig. 2E), defined as the number of nu- clei in myosin heavy chain (DSHB) positive cells divided by the total number of nuclei per surface area. These results indicate that PPP1R1BIncRNA has a conserved function in human myogenesis.

\section{Human PPP1R1B-IncRNA exhibited similar roles in myogenesis of human cardiomyocytes}

LncRNAs are known to have tissue specificity. To examine whether the human specific PPP1R1B-IncRNA affect myogenesis during cardiac muscle development, we used a humaninduced pluripotent stem cell-derived cardiomyocyte (hiPSC-CM) cell line. By silencing the PPP1R1B-IncRNA, myogenic differentiation of cardiomyocytes was impaired. The iPSCCMs also exhibited significantly less myosin expression and myofibrillar bundles following PPP1R1B-IncRNA siRNA treatment (Fig. 3A,B). Furthermore, PPP1R1BIncRNA knockdown led to significant diminution of the sizes of the differentiation aggregates, which are known as embryonic bodies (EBs). The EB density, defined as the total number of nuclei in EB divided by the surface EB area, was also decreased (Fig. 3C). These results further confirmed a conserved function for the human PPP1R1BIncRNA in promoting myogenesis in heart.

\section{Ppp1r1b-IncRNA positively regulates muscle-specific transcription factors and muscle structure genes}

To further characterize the function of Ppp1r1b-IncRNA in myogenesis, we examined the impact of its loss on mRNA expression of the muscle-specific transcription factors and muscle structure genes. It was known that the differentiation of skeletal muscle cells is governed by a group of four myogenic regulatory factors (MRFs) including MyoD, Myf5, Myogenin and MRF4 (Buckingham and Rigby 2014). MyoD is generally believed to act as a fate determination gene, while myogenin is essential for the terminal differentiation.

Our data revealed that mRNA levels of Myogenin, MyoD1, Tbx5, Dystrophin and Troponin T2, were significantly lower in the GapmeR treated C2C12 cells compared with the untreated group (Fig. 4A). Likewise, siRNA treated human myoblasts showed a significant decrease in mRNA expression of the same set of myogenic transcription factors and muscle structural genes (Fig. 4B). Furthermore, GapmeR mediated Ppp1r1p-IncRNA silencing in neonatal mouse heart tissue revealed a similar trend (Fig. 4C). 

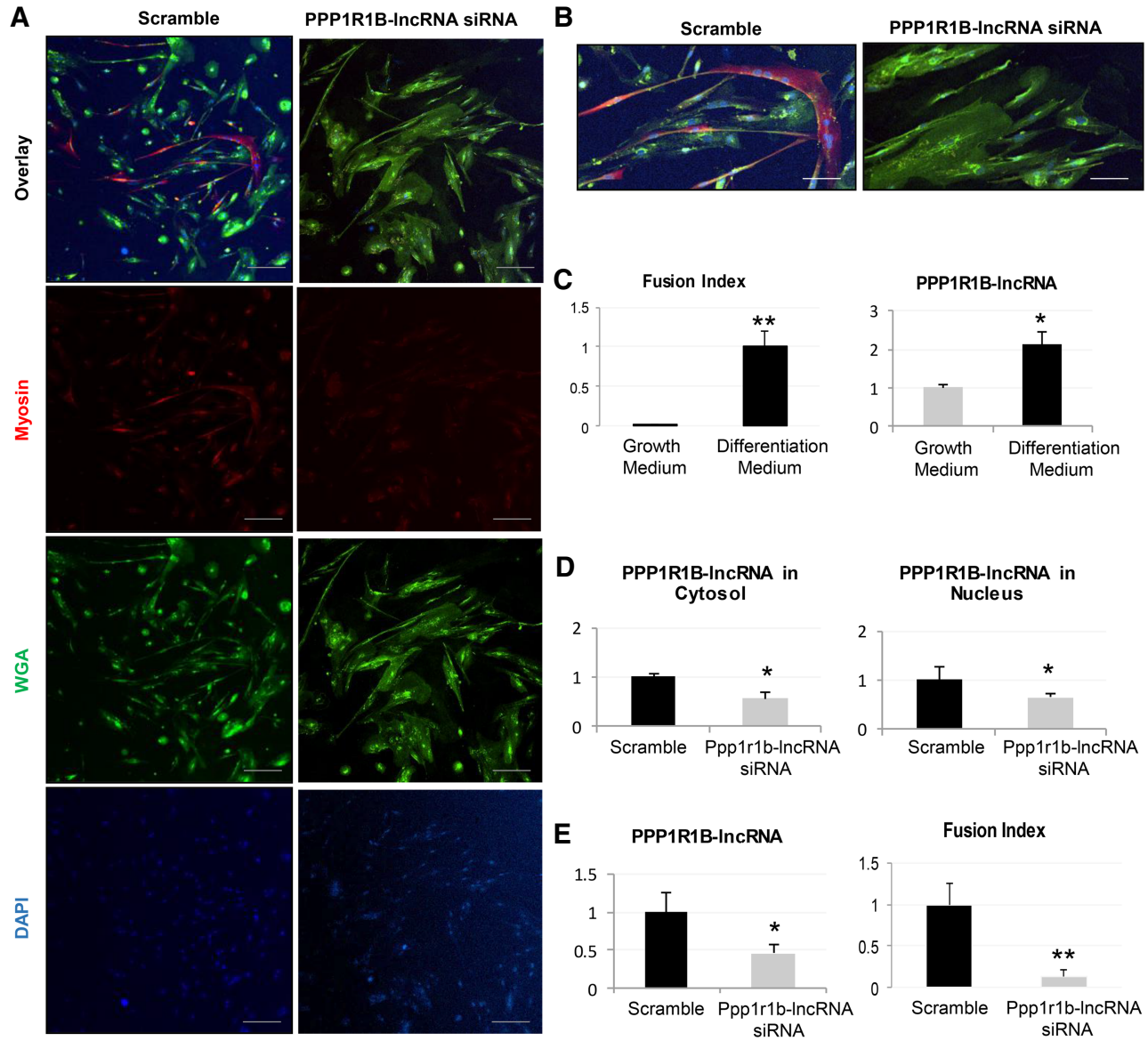

FIGURE 2. Human PP1R1B-IncRNA promotes differentiation of human skeletal myoblast. (A) Fluorescent microscope images depict inhibition of myogenesis of human skeletal myoblast (myosin positive cells) after PPP1R1B-IncRNA siRNA treatment. Scale bar, $100 \mu \mathrm{m}$. (B) Higher magnification images of cells presented in A. (C) Real-time PCR analysis and fusion index analysis of human myoblast before and after differentiation (625 nuclei counted for growth medium; 571 nuclei counted for differentiation medium). (D) RNA fractionation analysis of PPP1R1B-IncRNA down-regulated by siRNA. (E) Real-time PCR analysis and fusion index analysis of human myoblast after PPP1R1B-IncRNA siRNA treatment in differentiation medium (737 nuclei counted for Scramble; 760 nuclei counted for siRNA). $N=4$ biological replicates per condition; error bars, standard error of the mean; $\left(^{*}\right) P<0.05 ;\left(^{* *}\right) P<0.01$.

Moreover, siRNA treated hiPSC-CM also showed significant decrease in mRNA expression of muscle-specific transcription factors, including TBX5 and muscle structure genes (Fig. 4D). Taken together, the above findings suggest that Ppp1r1b-IncRNA modulates myogenesis by positively regulating myogenic transcription factors and structural genes of striated muscles development in both mouse and human myoblasts, cardiac progenitors, as well as neonatal mouse heart.

\section{Ppp1r1b-IncRNA negatively modulates histone methylation on the promoter of muscle-specific transcription factors}

It was reported that IncRNAs can precisely regulate skeletal muscle proliferation and differentiation by regulating H3K27me3 levels (Gan et al. 2019). To examine the epigenetic effects of Ppp1r1b-IncRNA on myogenesis associated gene expression, we performed ChIP-PCR analysis for
$\mathrm{H} 3 \mathrm{~K} 27 \mathrm{me} 3$ in $\mathrm{C} 2 \mathrm{C} 12$ cells. As shown in Figure $5 \mathrm{~A}, \mathrm{~B}$, the H3K27me3 levels on MyoD1 and Myogenin promoters were decreased in the differentiated myotubes compared to the undifferentiated myoblasts. After Ppp1r1b-IncRNA GapmeR treatment, the H3K27me3 levels were increased. These findings are consistent with the suppressed expression of these transcription regulators (Fig. 5C) and impaired differentiation after GapmeR treatment. From the above results, we deduced that Ppp1r1b-IncRNA positively regulates the expression of myogenic transcription factors by negatively modulating $\mathrm{H} 3 \mathrm{~K} 27 \mathrm{me} 3$ levels on their promoters.

\section{Ppp1r1b-IncRNA interacts with PRC2 core member, $\mathrm{EZH} 2$}

Polycomb repressive complex 2 (PRC2) possesses histone methyltransferase (HMT) activity with specificity for Lys 9 (K9) and Lys 27 (K27) of histone H3 (Kuzmichev et al. 
A
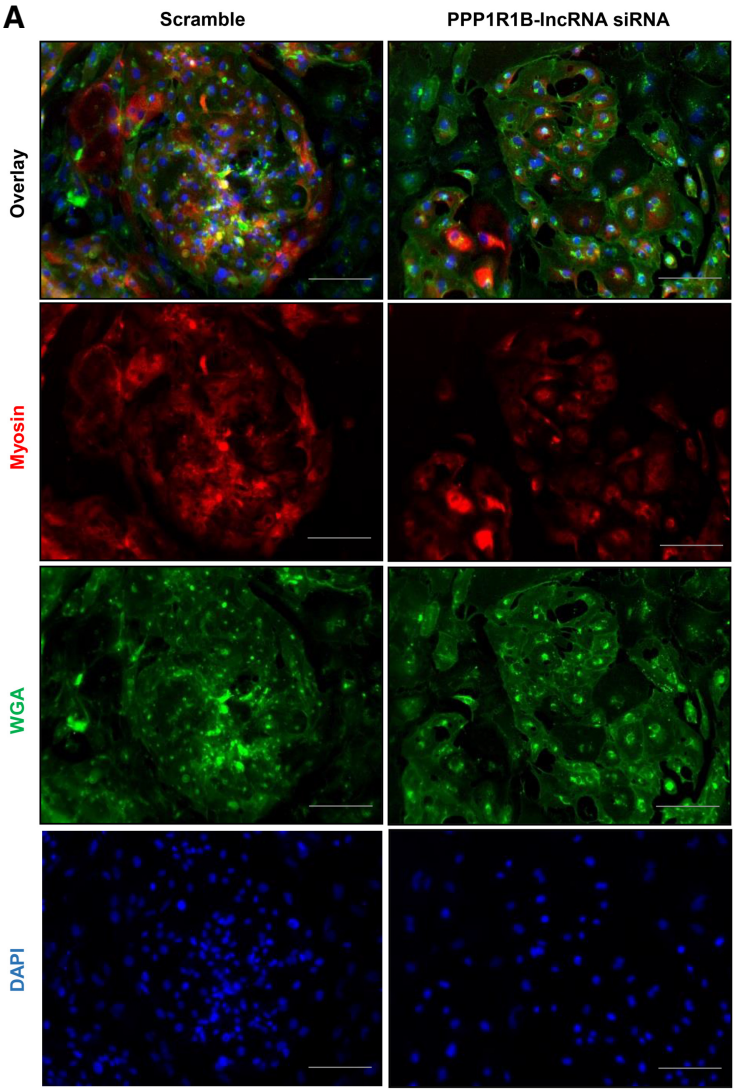

B
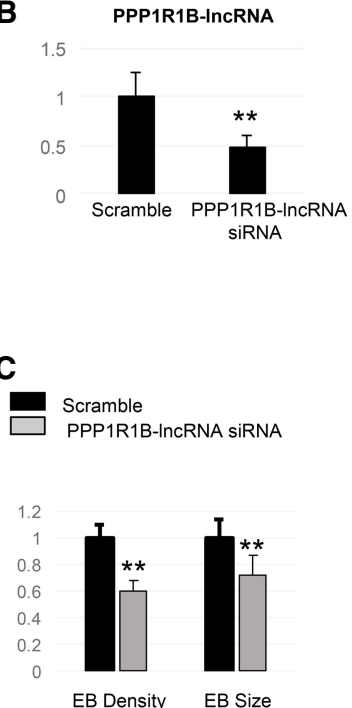

FIGURE 3. Human PPP1R1B-IncRNA promotes differentiation of human iPSC-derived cardiomyocyte (hiPSC-CM). (A) Microscope images depict the differentiation efficiency of human hiPSC-CM (Myosin positive cells) after PPP1R1B-IncRNA siRNA, or Scramble, treatment. (WGA) Wheat germ agglutinin. Scale bar, $100 \mu \mathrm{m}$. (B) Quantitative real-time PCR analysis of PPP1R1B-IncRNA expression after siRNA treatment. (C) Quantitative analysis depicts differentiation efficiency of hiPSC-CMs after siRNA treatment. (EB) Embryonic bodies. $N=4$ biological replicates per condition; error bars, standard error of the mean; $\left(^{*}\right) P<0.05 ;\left(^{* *}\right) P<0.01$.

2002). EZH2 is the functional enzymatic component of PRC2. To investigate the potential interaction between Ppp1r1b-IncRNA and PRC2, we performed an RNA pulldown assay using neonatal mouse heart tissue and demonstrated that Ezh2 protein co-precipitated with biotinylated Ppp1r1b-IncRNA (Fig. 6A). We next performed an RNA immunoprecipitation (RIP) assay using Ezh2 antibody, and detected an immune-complex of Ezh2 isolated from chromatin fractions with enriched Ppp1r1b-IncRNA (Fig. 6B). These results suggest that Ppp1r1b-IncRNA interacts directly with Ezh2 complex in the myocytes.

\section{Interaction of Ppp1r1b-IncRNA with MyoD1 and TBX5 promoters}

The association of PRC2 with chromatin is thought to be regulated by interaction with RNA. We showed that higher Ppp1r1b-IncRNA expression was correlated with lower H3K27me3 levels at the promoters of myogenic transcription factors (Fig. 5). We conducted chromatin isolation by
RNA purification (ChIRP) using mouse specific and human specific Ppp1r1bIncRNA probes, respectively. Promoter DNA of several myogenic regulators, including Myogenin, MyoD1 and Tbx5, was analyzed from the pulled RNA: DNA interactome (Fig. 7). Among them, MyoD1 and Tbx5 promoter DNA were significantly enriched in the Ppp1r1b-IncRNA probe isolated genomic DNA compared to a negative control probe (Fig. 7). However, Myogenin promoter DNA was not enriched, suggesting that MyoD1 is the most important myogenic factor regulated by Ppp1r1b-IncRNA. To further determine how Ppp1r1b-IncRNA inhibits histone methylation and correspondingly increases MyoD1 expression, we conducted a ChIP assay using EZH2 antibody, combined with Ppp1r1b-IncRNA silencing. The association of PRC2 with MyoD1 promoter DNA was negatively regulated by Ppp1r1b-IncRNA (Fig. 8). These results indicate that Ppp1r1bIncRNA inhibits H3K27 methylation at MyoD1 promoter regions by eviction of PRC2.

\section{DISCUSSION}

In this study, we show that Ppp1r1bIncRNA is necessary for normal myogenesis. By knocking down Ppp1r1bIncRNA, the expressions of myogenic transcription factors were significantly reduced. Although the IncRNAs are known to have species specificity and tissue specificity, Ppp1r1b-IncRNA appears to be a conserved transcriptional regulator in both human and mouse, and in both cardiac and skeletal muscle. It has been proven that developing skeletal muscle shares similar genetic network and common cell lineage with cardiac muscle during development. Du et al. (2008) found that noncardiac MHCs play a major role during the early sarcomere structure assembly in cardiomyocytes. Recently, it was revealed that cardiac Troponin I and $\mathrm{T}$ are also expressed in developing skeletal muscle (Sehnert et al. 2002; Clause et al. 2012). Thus, we can hypothesize that Ppp1r1b-IncRNA may modulate the initial differentiation via a common molecular mechanism, contributing to similar molecular features in the heart and skeletal muscle.

$\mathrm{H} 3 \mathrm{~K} 27 \mathrm{me} 3$ at promoter sites is a marker of transcriptional repression. It was reported that removal of this repressive marker is essential for the induction of cardiac 


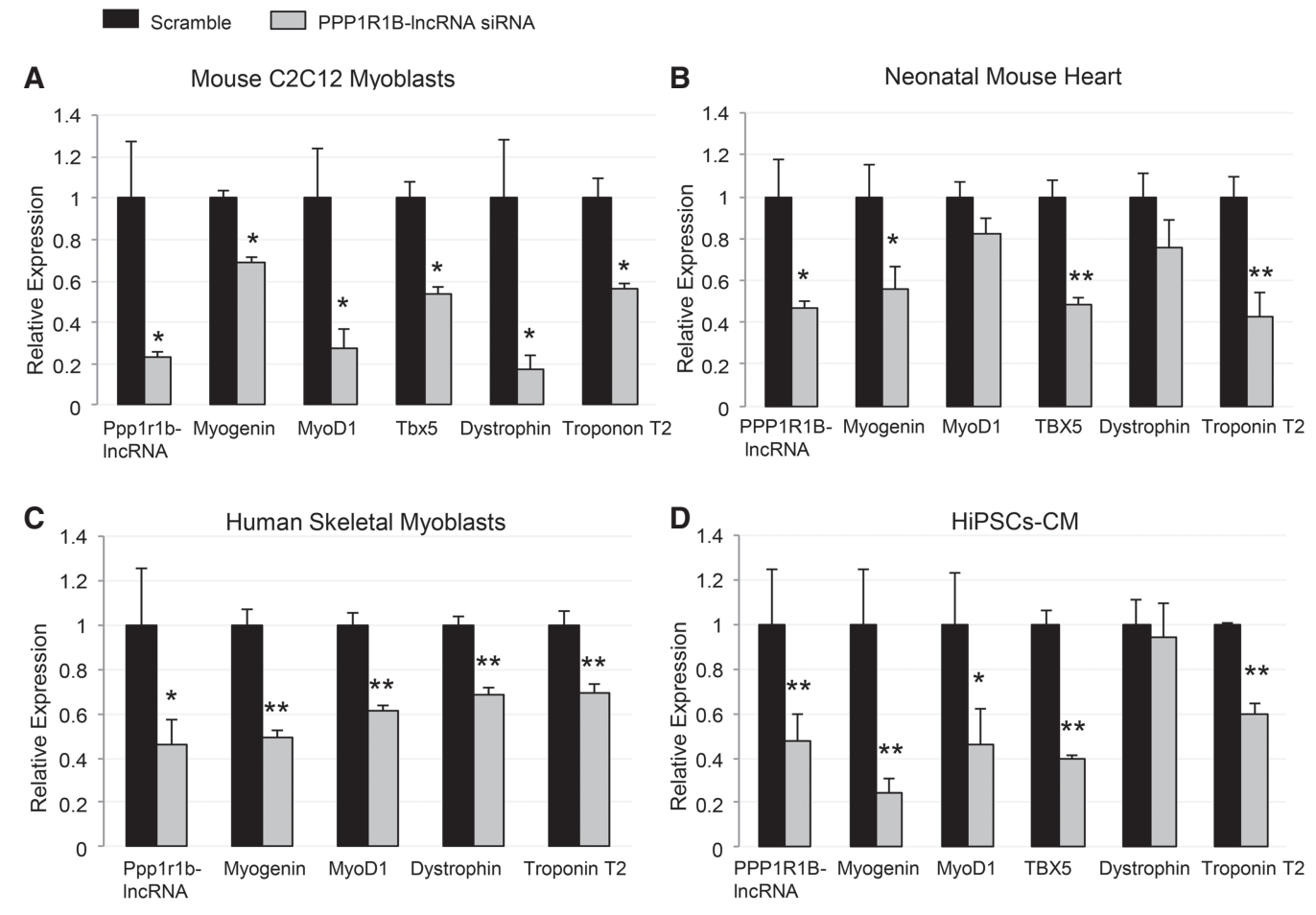

FIGURE 4. Ppp1r1b-ncRNA regulates expression of myogenic regulatory factors and sarcomere structural genes. Real-time PCR analysis of myogenic regulatory factors and sarcomeric structural genes after Ppp1r1b-IncRNA silencing in Mouse C2C12 myoblasts ( $A$ ), mouse heart tissue (B), human skeletal myoplasts $(C)$, and human-induced pluripotent stem cell-derived cardiomyocytes (hiPSCs-CM) $(D)$. $N=4$ biological replicates per condition. Error bars, standard error of the mean. $\left(^{*}\right) P<0.05 ;\left(^{* *}\right) P<0.01$.

reprogramming (Dal-Pra et al. 2017). The demethylation of H3K27me3 facilitates MyoD1-mediated myogenic differentiation (Akiyama et al. 2017). By examining the levels of trimethylated histone $\mathrm{H} 3$ of lysine 27 from GapmeR treated $\mathrm{C} 2 \mathrm{C} 12$ cell, our results showed that the silencing of Ppp1r1b-IncRNA increased the enrichment of H3K27me3 to predifferentiation levels, concurrent with a decrease in the expression of MyoD1 and Myogenin, at both mRNA and protein levels. Therefore, Ppp1r1b-IncRNA functions to promote MyoD1 and Myogenin transcription by negatively modulating the level of H3K27me3. However, the complete scope of Ppp1r1b-IncRNA targeted genes beyond MyoD1 and Myogenin during myogenesis remains to be fully characterized.

The EZH2 protein is the enzymatic component of PRC2 which catalyzes tri-methylation of histone $\mathrm{H} 3$ lysine 27. Using RIP, RNA pulldown and ChIRP analyses, we have demonstrated that Ppp1r1b-IncRNA interacts with EZH2, and binds on promoters of MyOD1 and TBX5. These results suggest that Ppp1r1b-IncRNA physically associates with PRC2 complex at promoters of myogenic transcription factors. The inhibition of H3K27me3 by Ppp1r1bIncRNA could be due to inhibition of PRC2 activity or eviction of PRC2 from chromatin by Ppp1r1b-IncRNA (Yan et al. 2019). It was also proposed that PRC2 recruitment or eviction is determined by the rate at which RNA is released from the locus. At a slow release rate, the majority of RNA remains attached to chromatin and thus PRC2RNA-chromatin are kept in close proximity. However, at high release rate, most of the RNA is freed from chromatin. Hence, PRC2 is titrated away by RNA from chromatin (Prasanth and Spector 2007). Our results propose that Ppp1r1b-IncRNA precludes PRC2 recruitment to MyoD1 promoter by competing for PRC2 binding.

In our study, we identified Ppp1r1b-IncRNA as a required regulator of myogenesis in striated, skeletal and cardiac muscle cells. It efficiently works on some myogenesis transcription factors such as MyoD1 and Tbx5 to initiate the differentiation of skeletal myoblasts and hiPSCsderived cardiomyocytes. In skeletal muscle, MyoD has been identified as a master regulator of differentiation (Davis et al. 1987). It was also reported that MyoD depletion leads to a dystrophy-associated cardiomyopathy (Megeney et al. 1999). Some types of muscular dystrophy are also associated with cardiomyopathy (Muntoni 2003). Progression of skeletal muscle damage is a significant contributing factor leading to development of cardiomyopathy (Megeney et al. 1999). Thus, we speculate that together with MyoD, Ppp1r1b-IncRNA may play a role in cardiac diseases progression. Further studies are needed to explore this potential role.

The cardiac transcription factor Tbx5 is a key regulator of heart development (Steimle and Moskowitz 2017). During early cardiac development, Tbx5 appears to act primarily 
A

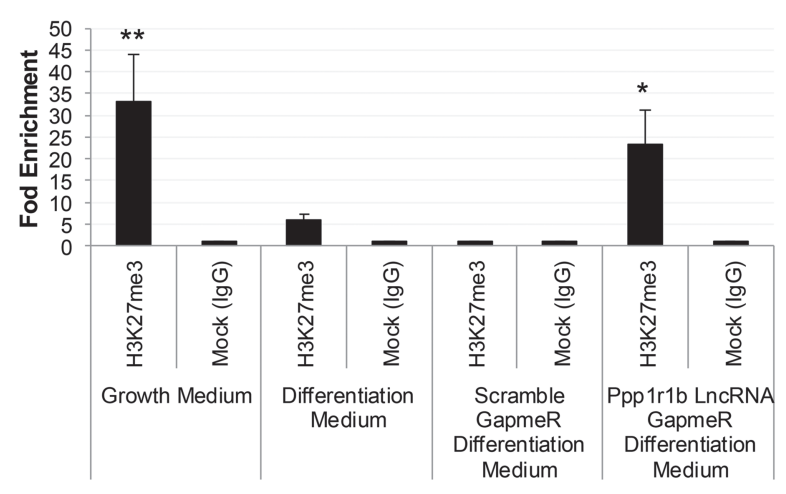

B

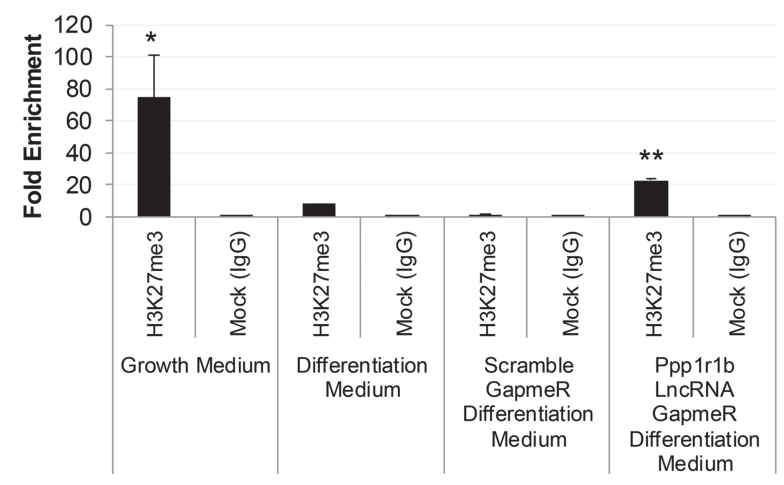

C Untreated Differentiation Medium

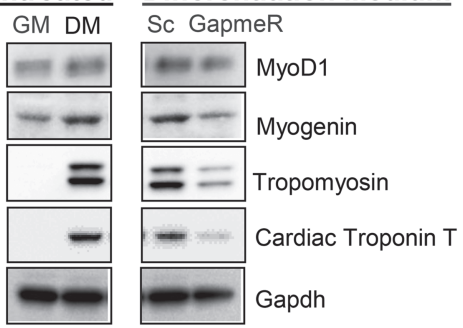

FIGURE 5. Ppp1r1b-IncRNA inhibits Histone 3 methylation at the promoter regions of myogenic regulatory factors. C2C12 myoblasts were cultured in growth medium (GM) or differentiation medium (DM) and treated by scramble GapmeR or Ppp1r1b-IncRNA GapmeR. After treatment, cells were subjected to ChIP analysis using anti H3K27me3 antibodies. The qPCR data are presented as fold enrichment to the background, indicating the abundance of histone modifications at the promoter regions of the myogenic genes. ( $A)$ H3K27me3 level on MyoD1 promoter. (B) H3K27me3 level on Myogenin promoter. (C) Western blot analysis of myogenic regulatory factors and sarcomeric structural proteins from C2C12. Gapdh was used as a loading control. (GM) Growth medium, (DM) differentiation medium. (*) $P<0.05 ;\left(^{* *}\right) P<0.01$.

as a transcriptional activator of genes associated with cardiomyocyte maturation and upstream of the morphological signals for septation. Human mutations in TBX5 cause congenital heart disease (CHD) (Baban et al. 2014; AlQattan and Abou Al-Shaar 2015), although the underlying mechanism is unknown. It is reported that Tbx5 is regulat- ed through microRNA dependent mechanisms (Wang et al. 2014) and may play a role in adult conduction defects and pathological remodeling in diseased hearts (Torrado et al. 2015). To date, there is no report about interaction between the Tbx5 promoter and IncRNA. Our results, for the first time, demonstrated that the newly identified Ppp1r1b-IncRNA is involved in Tbx5 regulation during cardiac myogenesis and development, although further studies will be needed to elucidate the role of Ppp1r1b-IncRNA in regulating Tbx 5 expression.

Our study has revealed the crucial roles of Ppp1r1bIncRNA in myogenesis control and potential molecular mechanisms. Heart formation encompasses an orchestrated series of molecular and cellular events, and thus even subtle alterations in this process can lead to serious cardiac disorders. On the other hand, cardiac muscle has limited proliferative capacity and regenerative therapies are highly in demand as a new treatment strategy. Our results may facilitate our understanding of the function of Ppp1r1bIncRNA and provide us with a promising therapeutic target for congenital heart disease and skeletal myopathy. However, we should acknowledge important limitations: (i) Although several models have demonstrated the molecular mechanisms involved in the IncRNA dependent inhibition of histone methylation (Wang et al. 2017), further studies are needed to elucidate the detailed mechanisms of Ppp1r1b-IncRNA function in the regulation of MyoD1 and Tbx5. In addition, the mechanism by which Ppp1r1bIncRNA regulates Myogenin expression remains to be investigated. (ii) While our results support a role for the Ppp1r1b-IncRNA during early stages of muscle and heart development, our in vivo studies are limited to temporal Ppp1r1b-IncRNA silencing during the neonatal period. A comprehensive in vivo characterization of the functional roles during development in transgenic mouse models is required. (iii) Examining the translational value of Ppp1r1b-IncRNA manipulation requires temporal and tissue specific in vivo delivery of Ppp1 1 1b-IncRNA in disease models of muscular dystrophy, cardiomyopathy, and congenital heart disease.

\section{MATERIALS AND METHODS}

Extended materials and their sources are available in the Supplemental Information Appendix.

\section{Cell lines and human heart tissue}

C2C12 and Human skeletal muscle myoblast cell lines were obtained from ATCC, and ZenBio. Human iPSC-derived cardiomyocytes (SCVI480-CM) were obtained from Joseph C. Wu MD, PhD at the Stanford Cardiovascular Institute (CVI), Stanford; CA. Cells were cultured according to protocols established at the CVI (Wu et al. 2019). Human heart specimens were obtained from the UCLA-Translational Pathology Core Laboratory (TPCL) Core and 

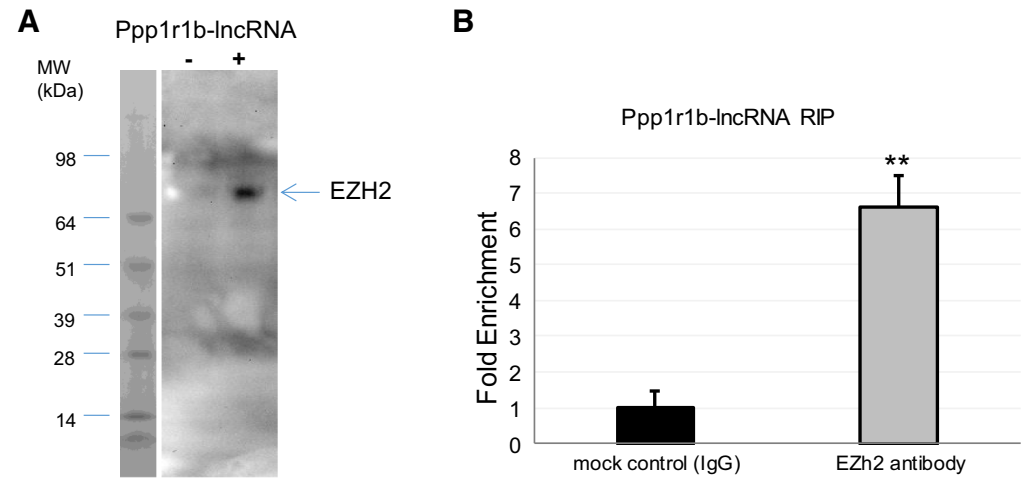

FIGURE 6. Ppp1r1b-IncRNA binds EZH2. (A) RNA pull-down assays were carried out by incubating cell lysate from neonatal mouse heart tissue with biotinylated in vitro transcribed Ppp1r1b-IncRNA. Subsequent western blotting analysis revealed EZH2 in the isolated RNAprotein complex. (B) RNA immunoprecipitation (RIP) demonstrated that Ppp1r1b-IncRNA was immunoprecipitated together with $\mathrm{EZH} 2$ in neonatal mouse heart tissue. LncRNA was detected using real-time PCR. $\left(^{*}\right) P<0.05$.
Purification Kit (Norgen Biotek) following the manufacturer's manual.

\section{Real-time PCR analysis}

Total RNA was isolated from the cultured cells or tissue using RNeasy Mini Kit (Qiagen). The reverse transcription reaction was performed with $1 \mu \mathrm{g}$ total RNA and the SuperScript IV First-Strand Synthesis System (Thermo Fisher Scientific), and the real-time PCR reactions were performed by using iTaq Universial SYBR Green Supermix from BioRad. Primers were prepared by Invitrogen. (Supplemental Information Appendix, Table S1). Relative expression value was calculated using the comparative threshold cycle $(\Delta \Delta C T)$ method. the UCLA Congenital Heart Defect-BioCore (Touma et al. 2017). Human studies were conducted in accordance with regulation of the University of California Los Angeles Institutional Review Board.

\section{Mouse injection and in vivo study}

All animal-related experimental protocols were approved by the University of California Los Angeles Animal Care and Use Committee (ACUC). Wild-type C57BL/6 mice were purchased from Charles River laboratory. Neonatal mice were used for Ppp1r1b-IncRNA GapmeR injection in vivo. C57BL/6 neonatal mouse pups were intravenously injected with $1.2 \mathrm{nmol} / \mathrm{g}$ body weight GapmeR at P1, P3, and P5. Mice were sacrificed at P7 and heart tissues were collected. Experimental protocols were approved by the University of California Los Angeles Animal Care and Use Committee.

\section{Cell transfection}

Transient transfection of cells with GapmeR (Qiagen) and siRNA (Bioland Scientific) was performed in 12-well plates using Lipofectamine 2000 reagent or RNAiMAX (Life Technologies). Mouse Ppp1r1b-IncRNA GapmeR $(50$ pmol/ well) was transfected into $\mathrm{C} 2 \mathrm{C} 12$ cells cultured in differentiation media. Human PPP1R1B-IncRNA siRNA (30 pmol/well) was transfected into human skeletal muscle myoblast and human iPSC-CM cultured in differentiation media.

\section{Isolation of cytoplasmic and nuclear RNA}

Cytoplasmic and nuclear RNA were isolated with the Cytoplasmic and Nuclear RNA

\section{Western blotting}

Cell extracts from cultured cells or tissue were fractionated by SDS-PAGE. The iBlot 2 Dry Blotting System and iBind Western System (Thermo Fisher Scientific) were used to transfer proteins to a membrane for analyzing with antibodies. Antibodies were listed in Supplemental Information Appendix, Table S2.

\section{Immunofluorescence staining}

Cells were fixed in $4 \%(v / v)$ formaldehyde in PBS for $20 \mathrm{~min}$. After rinsing with PBS, the cells were incubated with $0.1 \%$ TritonX-100 in PBS for $15 \mathrm{~min}$ at room temperature and rinsed. After blocking in PBS containing 10\% Bovine Serum Albumin for $1 \mathrm{~h}$, the cells were incubated overnight with primary antibody and then appropriate AlexaFluor conjugated secondary antibodies for $1 \mathrm{~h}$. Cell nuclei were eventually counterstained by DAPI. Images were recorded on a Nikon confocal microscope and an echo revolve microscope (San Diego, CA). Fusion index was defined as the

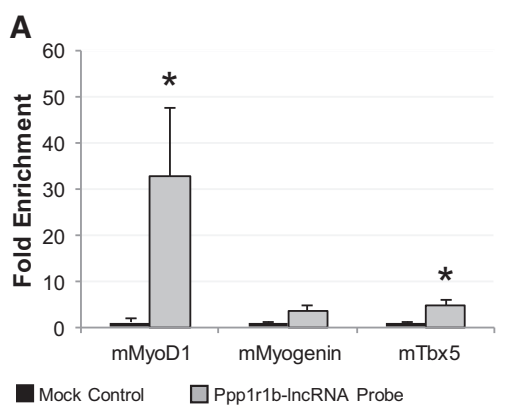

B

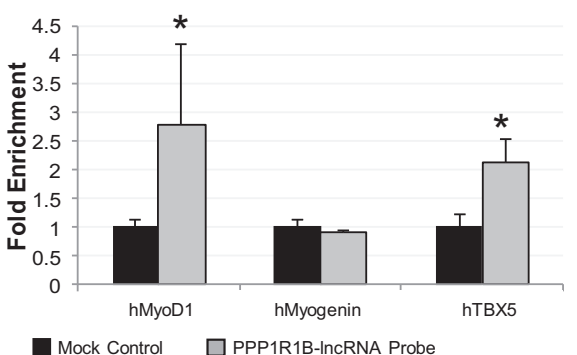

FIGURE 7. Ppp1r1b-IncRNA binds to MyoD1, Myogenin and Tbx5 promoter region. Chromatin isolation by RNA purification (ChIRP) assays were carried out by incubating cell lysate from mouse neonatal heart tissue and human infantile heart tissue with biotinylated in vitro synthesized Ppp1r1b-IncRNA probes. Promoter DNAs were detected by real-time PCR. (A) Interactions of Ppp1r1b-IncRNA with MyoD1, Myogenin, and Tbx5 promoter DNA in mouse. (B) Interactions of Ppp1r1b-IncRNA with MyoD1, Myogenin, and Tbx5 promoter DNA in human. $\left({ }^{*}\right) P<0.05$. 


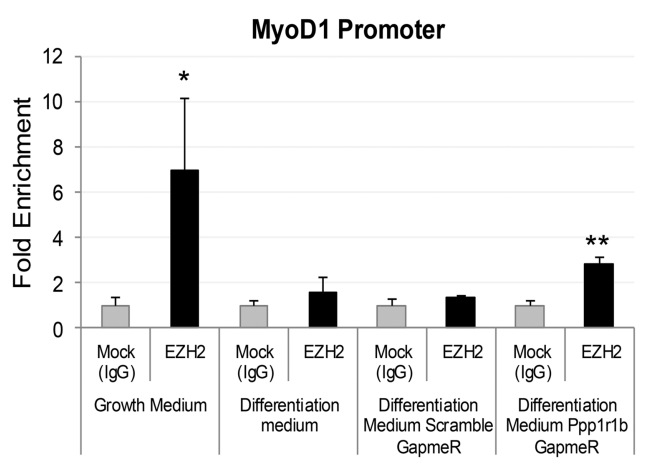

FIGURE 8. Ppp1r1b-IncRNA Inhibits EZH2 Binding at the MyoD1 promoter region. $\mathrm{C} 2 \mathrm{C} 12$ myoblasts were cultured in growth and differentiation medium (DM) treated with scramble GapmeR and Ppp1r1b-IncRNA GapmeR. Treated C2C12 cells were subjected to ChIP analysis using anti-EZH2 antibodies. The qPCR data are presented as fold enrichment to the background, indicating the EZH2 abundance at the MyoD1 promoter region. $\left(^{*}\right) P<0.05 ;\left(^{* *}\right) P<0.01$.

number of nuclei in myosin heavy chain (DSHB) positive cells divided by the total number of nuclei. EB density was defined as the total number of nuclei in EB divided by the EB area.

\section{In vitro expression and translation assay}

The full-length mouse and human IncRNA cDNA was cloned into PCMV Sport 6.1 vector, which contains a T7 or a SP6 promoter. The Transend nonradioactive system was used to incorporate biotinylated lysine into nascent proteins. After the in vitro TNT reaction, the reaction was terminated by adding SDS loading buffer and incubation at $95^{\circ} \mathrm{C}$ for $5 \mathrm{~min}$. After SDS-PAGE and electro blotting, the biotinylated proteins were visualized by binding Streptavidin-HRP and chemiluminesent detection.

\section{Chromatin immunoprecipitation (ChIP)}

Undifferentiated and differentiated $\mathrm{C} 2 \mathrm{C} 12$ as well as Ppp1r1bIncRNA GapmeR treated C2C12 cells were exposed to $1 \%$ formaldehyde, and chromatin was fragmented by sonication. Immunoprecipitation was performed using IgG (negative control) or with the primary antibodies for $\mathrm{H} 3 \mathrm{~K} 27 \mathrm{me} 3$ or EZH2. DNA was purified from the immunoprecipitates using the PCR purification kit (Qiagen). Subsequently, real-time PCR was performed to amplify the promoter region DNA of Myogenin and MyoD1. Primers were prepared by Invitrogen (Supplemental Information Appendix, Table S1). Data were analyzed using fold enrichment method.

\section{RNA pulldown}

The assay was performed with P0 C57BL/6 mouse hearts. T7 RNA polymerase kit (Promega) and Biotin RNA labeling mix (Roche) were used for preparation of biotin-labeled Ppp1r1bIncRNA. After incubated biotinylated RNA with cell extract at $4^{\circ} \mathrm{C}$, the washed Dynabeads M280 Streptavidin (Thermo Fisher Scientific) was added to the pulldown mixture. After $1 \mathrm{~h}$ incubation at $4^{\circ} \mathrm{C}$ for $1 \mathrm{~h}$, the beads were collected by using the magnet- ic apparatus and washed with the binding buffer. Proteins that were co-precipitated were eluted in the SDS sample buffer and fractionated in 8\%-16\% gradient SDS-PAGE for western analysis with EZH2 antibody (Supplemental Information Appendix, Table S2).

\section{RNA immunoprecipitation (RIP)}

RNA immunoprecipitation experiments were performed with P1 neonatal mouse heart tissue. Tissue lysate was cross-linked by $1 \%$ formaldehyde and lysed by sonication. After EZH2 antibody (Supplemental Information Appendix, Table S2) was added to cell lysate and incubated overnight at $4^{\circ} \mathrm{C}$, Protein $A / G$ beads (Thermo Scientific) were added and incubated for $1 \mathrm{~h}$ at $4^{\circ} \mathrm{C}$ with gentle rotation. After washed off unbound material, RNAs bound to immunoprecipitated EZH2 were isolated and qRTPCR was performed using Ppp1r1b-IncRNA primers (Supplemental Information Appendix, Table S1).

\section{Chromatin isolation by RNA purification (CHIRP)}

The Magna CHIRP RNA interactome kit (EMD Millipore Corp.) was used. Assays were performed per manufacturer's protocol. Capture probes were designed to target Ppp1r1b-IncRNA and prepared at $50 \mu \mathrm{M}$ total oligo concentration. P1 mouse heart tissue and human fetal heart tissue were cross-linked in 1\% glutaraldehyde/PBS and sonicated to shear DNA. Probes were added to cell lysate and incubated overnight for hybridization. After hybridization, Streptavidin magnetic beads were added and incubated for an additional $30 \mathrm{~min}$. After washing, beads were incubated with RNase $A$ and RNase $H$ in DNA elution buffer. The eluted sample was then subjected to protease $K$ treatment, DNA isolation and quantitative PCR analysis. Primers used for real-time PCR were listed in Supplemental Information Appendix, Table S1.

\section{Statistical analysis}

Quantified results are presented as mean \pm SEM. Comparisons between groups were evaluated using analysis of variance or the Student's t-test; $P \leq 0.05$ was considered significant unless specified otherwise.

\section{DATA DEPOSITION}

Gene expression data will be deposited within the Gene Expression Omnibus repository (www.ncbi.nlm.nih.gov/geo) under Neonatal Heart Maturation SuperSeries GSE85728 (http:// www.ncbi.nlm.nih.gov/geo/query/acc.cgi?acc=GSE85728). All unique materials, resources, and reagents are available on request by qualified researchers for their own use. Generated plasmid constructs will be deposited in addgene.

\section{SUPPLEMENTAL MATERIAL}

Supplemental material is available for this article. 


\section{ACKNOWLEDGMENTS}

The human iPSC lines were obtained from Joseph C. Wu, MD $\mathrm{PhD}$ at the Stanford Cardiovascular Institute funded by National Institutes of Health (NIH) R24HL117756. The human tissue specimens were provided by the UCLA TPLC and the UCLA Congenital Heart Defects-BioCore funded by W81XWH-18-1-0164; we acknowledge the support of the Clinical Genomics Center at the UCLA Institute of Precision Health, and the Animal Physiology Core at the UCLA Division of Molecular Medicine. This work was supported by grants from the American Heart Association Career Development award 18CDA34110414; the Department of Defense-Congressionally Directed Medical Research Programs W81XWH-18-1-0164; the UCLA-Children's Discovery Institute and Today and Tomorrow Children's Fund; the David Geffen School of Medicine Cardiovascular Theme Research Innovation Seed grant to M.T., and the National Heart, Lung, and Blood Institute (1R56HL146738-01).

Author contributions: The research was designed by X.K. and M.T. and conducted by X.K., Y.Z., and M.T. G.V.A. and S.F.N. contributed new reagents/analytic tools. Data were analyzed by X.K., Y.Z., and M.T. X.K. and M.T. wrote the manuscript.

Received October 17, 2019; accepted January 13, 2020.

\section{REFERENCES}

Adam RC, Fuchs E. 2016. The yin and yang of chromatin dynamics in stem cell fate selection. Trends Genet 32: 89-100. doi:10.1016/j .tig.2015.11.002

Akiyama T, Wakabayashi S, Soma A, Sato S, Nakatake Y, Oda M, Murakami M, Sakota M, Chikazawa-Nohtomi N, Ko SBH, et al. 2017. Epigenetic manipulation facilitates the generation of skeletal muscle cells from pluripotent stem cells. Stem Cells Int 2017: 7215010. doi:10.1155/2017/7215010

Aloia L, Di Stefano B, Di Croce L. 2013. Polycomb complexes in stem cells and embryonic development. Development 140: 25252534. doi:10.1242/dev.091553

Al-Qattan MM, Abou Al-Shaar H. 2015. Molecular basis of the clinical features of Holt-Oram syndrome resulting from missense and extended protein mutations of the TBX5 gene as well as TBX5 intragenic duplications. Gene 560: 129-136. doi:10.1016/j.gene.2015 .02 .017

Baban A, Postma AV, Marini M, Trocchio G, Santilli A, Pelegrini M, Sirleto P, Lerone M, Albanese SB, Barnett P, et al. 2014. Identification of TBX5 mutations in a series of 94 patients with Tetralogy of Fallot. Am J Med Genet A 164a: 3100-3107. doi:10 .1002/ajmg.a.36783

Beltran M, Yates CM, Skalska L, Dawson M, Reis FP, Viiri K, Fisher CL, Sibley CR, Foster BM, Bartke T, et al. 2016. The interaction of PRC2 with RNA or chromatin is mutually antagonistic. Genome Res 26: 896-907. doi:10.1101/gr.197632.115

Buckingham M, Rigby PW. 2014. Gene regulatory networks and transcriptional mechanisms that control myogenesis. Dev Cell 28: 225-238. doi:10.1016/j.devcel.2013.12.020

Clause KC, Tchao J, Powell MC, Liu LJ, Huard J, Keller BB, Tobita K. 2012. Developing cardiac and skeletal muscle share fast-skeletal myosin heavy chain and cardiac troponin-I expression. PLoS One 7: e40725. doi:10.1371/journal.pone.0040725

Dal-Pra S, Hodgkinson CP, Mirotsou M, Kirste I, Dzau VJ. 2017. Demethylation of H3K27 is essential for the induction of direct cardiac reprogramming by miR combo. Circ Res 120: 1403-1413. doi:10.1161/CIRCRESAHA.116.308741
Davis RL, Weintraub H, Lassar AB. 1987. Expression of a single transfected cDNA converts fibroblasts to myoblasts. Cell 51: 987-1000. doi:10.1016/0092-8674(87)90585-X

Du A, Sanger JM, Sanger JW. 2008. Cardiac myofibrillogenesis inside intact embryonic hearts. Dev Biol 318: 236-246. doi:10.1016/j .ydbio.2008.03.011

Gan YM, Zhou J, Quan R, Hong LJ, Li ZC, Zheng EQ, Liu W, Wu ZF, Cai GY, Gu T. 2019. [Histone H3K27me3 in the regulation of skeletal muscle development]. Yi Chuan 41: 285-292.

Garry DJ, Olson EN. 2006. A common progenitor at the heart of development. Cell 127: 1101-1104. doi:10.1016/j.cell.2006.11 .031

Hassan N, Tchao J, Tobita K. 2014. Concise review: skeletal muscle stem cells and cardiac lineage: potential for heart repair. Stem Cells Transl Med 3: 183-193. doi:10.5966/sctm.2013-0122

Hernández-Hernández JM, García-González EG, Brun CE, Rudnicki MA. 2017. The myogenic regulatory factors, determinants of muscle development, cell identity and regeneration. Semin Cell Dev Biol 72: 10-18. doi:10.1016/j.semcdb.2017.11 .010

Karner HM, Webb CH, Carmona S, Liu Y, Lin B, Erhard M, Chan D, Baldi P, Spitale RC, Sun S. 2019. Functional conservation of IncRNA JPX despite sequence and structural divergence. J Mol Biol. doi:10.1016/j.jmb.2019.09.002

Kouzarides T. 2007. Chromatin modifications and their function. Cell 128: 693-705. doi:10.1016/j.cell.2007.02.005

Kuzmichev A, Nishioka K, Erdjument-Bromage H, Tempst P, Reinberg D. 2002. Histone methyltransferase activity associated with a human multiprotein complex containing the Enhancer of Zeste protein. Genes Dev 16: 2893-2905. doi:10.1101/gad .1035902

Liu Z, Chen O, Zheng M, Wang L, Zhou Y, Yin C, Liu J, Qian L. 2016. Re-patterning of H3K27me3, H3K4me3 and DNA methylation during fibroblast conversion into induced cardiomyocytes. Stem Cell Res 16: 507-518. doi:10.1016/j.scr.2016.02.037

Megeney LA, Kablar B, Perry RL, Ying C, May L, Rudnicki MA. 1999. Severe cardiomyopathy in mice lacking dystrophin and MyoD. Proc Natl Acad Sci 96: 220-225. doi:10.1073/pnas.96.1 .220

Muntoni F. 2003. Cardiomyopathy in muscular dystrophies. Curr Opin Neurol 16: 577-583. doi:10.1097/00019052-20031000000003

Olson EN. 2006. Gene regulatory networks in the evolution and development of the heart. Science 313: 1922-1927. doi:10.1126/sci ence.1132292

Ounzain S, Crippa S, Pedrazzini T. 2013. Small and long non-coding RNAs in cardiac homeostasis and regeneration. Biochim Biophys Acta 1833: 923-933. doi:10.1016/j.bbamcr.2012.08.010

Paralkar VR, Mishra T, Luan J, Yao Y, Kossenkov AV, Anderson SM, Dunagin M, Pimkin M, Gore M, Sun D, et al. 2014. Lineage and species-specific long noncoding RNAs during erythro-megakaryocytic development. Blood 123: 1927-1937. doi:10.1182/blood2013-12-544494

Prasanth KV, Spector DL. 2007. Eukaryotic regulatory RNAs: an answer to the 'genome complexity' conundrum. Genes Dev 21: 11-42. doi:10.1101/gad.1484207

Schuettengruber B, Cavalli G. 2009. Recruitment of polycomb group complexes and their role in the dynamic regulation of cell fate choice. Development 136: 3531-3542. doi:10.1242/dev.033902

Sehnert AJ, Huq A, Weinstein BM, Walker C, Fishman M, Stainier DY. 2002. Cardiac troponin $T$ is essential in sarcomere assembly and cardiac contractility. Nat Genet 31: 106-110. doi:10.1038/ng875

Smola MJ, Christy TW, Inoue K, Nicholson CO, Friedersdorf M, Keene JD, Lee DM, Calabrese JM, Weeks KM. 2016. SHAPE reveals transcript-wide interactions, complex structural domains, 
and protein interactions across the Xist IncRNA in living cells. Proc Natl Acad Sci 113: 10322-10327. doi:10.1073/pnas.1600008113

Steimle JD, Moskowitz IP. 2017. TBX5: a key regulator of heart development. Curr Top Dev Biol 122: 195-221. doi:10.1016/bs.ctdb .2016 .08 .008

Su M, Xiao Y, Tang J, Wu J, Ma J, Tian B, Zhou Y, Wang H, Yang D, Liao QJ, et al. 2018. Role of IncRNA and EZH2 interaction/regulatory network in lung cancer. J Cancer 9: 4156-4165. doi:10.7150/ jca. 27098

Torrado M, Franco D, Lozano-Velasco $E$, Hernández-Torres $F$, Calviño R, Aldama G, Centeno A, Castro-Beiras A, Mikhailov A. 2015. A microRNA-transcription factor blueprint for early atrial arrhythmogenic remodeling. Biomed Res Int 2015: 263151. doi:10.1155/2015/263151

Touma M, Kang $X$, Zhao $Y$, Cass AA, Gao F, Biniwale $R$, Coppola G, Xiao X, Reemtsen B, Wang Y. 2016. Decoding the long noncoding RNA during cardiac maturation: a roadmap for functional discovery. Circ Cardiovas Genet 9: 395-407. doi:10 $.1161 /$ CIRCGENETICS.115.001363
Touma M, Reemtsen B, Halnon N, Alejos J, Finn JP, Nelson SF, Wang Y. 2017. A path to implement precision child health cardiovascular medicine. Front Cardiovasc Med 4: 36. doi:10.3389/fcvm .2017 .00036

Wang $F$, Yang XY, Zhao JY, Yu LW, Zhang P, Duan WY, Chong M, Gui YH. 2014. MiR-10a and miR-10b target the $3^{\prime}$-untranslated region of TBX5 to repress its expression. Pediatr Cardiol 35: 1072 1079. doi:10.1007/s00246-014-0901-y

Wang X, Paucek RD, Gooding AR, Brown ZZ, Ge EJ, Muir TW, Cech TR. 2017. Molecular analysis of PRC2 recruitment to DNA in chromatin and its inhibition by RNA. Nat Struct Mol Biol 24: 1028-1038. doi:10.1038/nsmb.3487

Wu JC, Woo YJ, Mayerle M, Harrington RA, Quertermous T. 2019. Stanford Cardiovascular Institute. Circ Res 124: 1420-1424. doi:10.1161/CIRCRESAHA.119.310761

Yan J, Dutta B, Hee YT, Chng WJ. 2019. Towards understanding of PRC2 binding to RNA. RNA Biol 16: 176-184. doi:10.1080/ 15476286.2019.1565283 

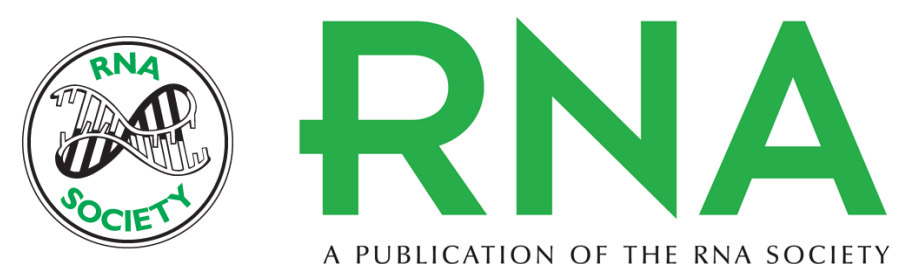

A PUBLICATION OF THE RNA SOCIETY

\section{Ppp1r1b-IncRNA inhibits PRC2 at myogenic regulatory genes to promote cardiac and skeletal muscle development in mouse and human}

Xuedong Kang, Yan Zhao, Glen Van Arsdell, et al.

RNA 2020 26: 481-491 originally published online January 17, 2020

Access the most recent version at doi:10.1261/rna.073692.119

Supplemental Material

References

Open Access

Creative Commons License

Email Alerting Service
http://rnajournal.cshlp.org/content/suppl/2020/01/17/rna.073692.119.DC1

This article cites 37 articles, 11 of which can be accessed free at: http://rnajournal.cshlp.org/content/26/4/481.full.html\#ref-list-1

Freely available online through the RNA Open Access option.

This article, published in $R N A$, is available under a Creative Commons License (Attribution 4.0 International), as described at http://creativecommons.org/licenses/by/4.0/.

Receive free email alerts when new articles cite this article - sign up in the box at the top right corner of the article or click here.

To subscribe to $R N A$ go to:

http://rnajournal.cshlp.org/subscriptions 